\title{
Design of a High-Energy and High-Resolution detector for X-ray computed tomography
}

\author{
Maëva Maulin ${ }^{1}$, Daniel Eck ${ }^{1}$, Nicolas Estre ${ }^{1}$, Emmanuel Payan ${ }^{1}$, Alix Sardet ${ }^{1}$, David Tisseur ${ }^{2}$, Grégoire Kessedjian ${ }^{2}$ \\ ${ }^{1}$ CEA, DES, IRESNE, DTN, Cadarache F-13108 Saint-Paul-lez-Durance, France. \\ ${ }^{2}$ CEA, DES, IRESNE, DER, Cadarache F-13108 Saint-Paul-lez-Durance, France. \\ Maeva.maulin@cea.fr
}

\begin{abstract}
The Nuclear Measurement Laboratory (LMN) at CEA Cadarache in France is developing a high-energy tomograph reaching energies up to $21 \mathrm{MeV}$ with high dose rates. It allows performing tomographies on massive objects (5 tons, $140 \mathrm{~cm}$ diameter) with a multimillimetric spatial resolution. For the control of absence of cracks, bubbles or defects in the concrete coating of some CEA waste drums, the laboratory needs a "high-resolution" version of this tomograph. The purpose of this study is to design an imaging detector able to provide a high spatial resolution on large objects with high dose resistance and scanning times of a few hours. To achieve this design, several steps are necessary and will be presented:
\end{abstract}

- A first step consists in characterizing the current detection elements of the tomograph, such as the camera and its lens with experimental measurements. This enables to compare performance of these elements with that available on the market and to consider a replacement.

- Then, the scintillator is selected according to experimental measurements on few scintillator types and a state of the art.

- Next, the detector configuration is optimized to achieve the higher spatial resolution.

Throughout these steps, we present the design of a highresolution detector with a spatial resolution around $300 \mu \mathrm{m}$ for a $10 \%$ contrast .

Keywords - Linear electron accelerator, High-energy computed tomography, High-resolution computed tomography, $\mathrm{X}$-ray detector

\section{INTRODUCTION}

$\mathrm{T}_{\mathrm{c}}$ HE Nuclear Measurement Laboratory (LMN) at CEA Cadarache has an imaging system currently implemented in the CHICADE experimental platform (Fig. 1) used to perform radiographic and tomographic analyses on large objects ( 5 tons, $140 \mathrm{~cm}$ diameter) with multimillimetric spatial resolution (recently measured around $2.1 \mathrm{~mm}$, obtained with (1)). This system, whose geometry is represented in Fig. 2, is composed of a high-energy photon source generated by the SATURNE accelerator which can reach energies up to $21 \mathrm{MeV}$ with high dose rates (60 Gy/min) [1]. The detector is composed of a scintillator and a sCMOS camera coupled to a lens, kept out of the primary irradiation beam with an angle-deflecting mirror. The objects to be scanned are placed between the source and the detector on a mechanical bench.

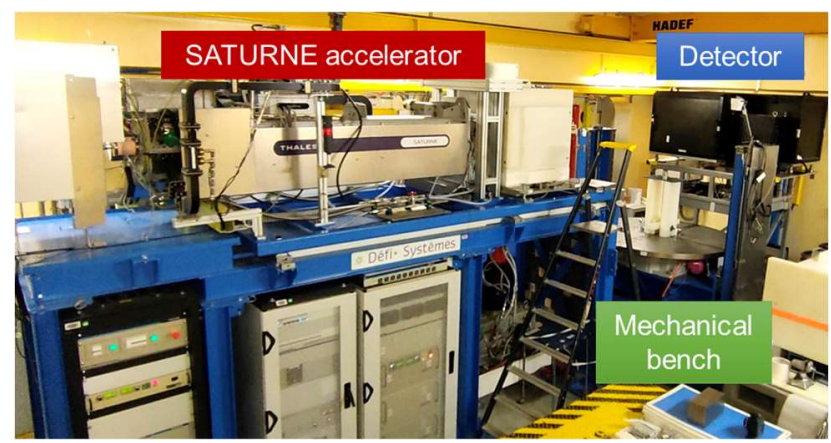

Fig. 1. Panoramic view of the accelerator SATURNE in the CHICADE platform (July 2019)

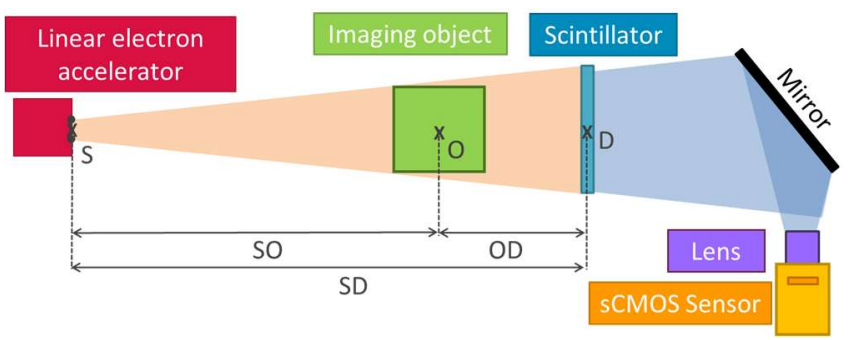

Fig. 2. Diagram of the tomograph geometry

The laboratory needs a "high-resolution" version of this tomograph to check that there are no cracks, bubbles or defects in the concrete coating of some CEA waste drums. Moreover, it can be used for non-destructive testing of metal pieces produced by additive manufacturing, such as specific heat exchangers.

Several types of high energy detectors enabling to obtain high resolution are commercially available:

Linear detectors producing one-dimensional images [2]. The main advantage of using this type of detector is the significant reduction of the scattered radiation, which allowing to obtain higher contrast and high resolution images. However, the linear technique using a small part of the X-rays field requires high scan times.

Flat panel detectors (FPD) integrate in the same structure a scintillator that converts X-rays into light (indirect conversion), which is read by a thin film transistor (TFT) [3]. This type of detector is quickly set up and allows short scan times with good spatial resolution, but their compact structure are rapidly damaged by the X-ray beam. 
- The X-ray imaging detectors made of a scintillator coupled via a mirror to a sCMOS camera (Fig. 2) have the advantage of being easily adaptable to system expectations and produced with large surface areas. The different elements can be adapted to the high resolution. However, due to their massive geometry and the optimization of the different elements to be realized, they can be difficult to implement compared to a flat panel detector.

The purpose of this study is to design an imaging detector made of a scintillator coupled to a sCMOS camera able to provide a high spatial resolution on large objects with high dose resistance and scanning times of a few hours. This corresponds to a resolution performance increase of one decade compared to the current tomograph.

To achieve this design, five main steps were necessary.

\section{STEP 1: OPTICAL ELEMENTS CHARACTERIZATION}

During the first step, the blur related to the different current detection elements are evaluated using experimental measurements on an optical bench.

The camera currently used on the tomograph is a Zyla camera from Andor [4] coupled with a HyperPrime lens [5]. We also performed tests on the Marana camera from Andor comporting a larger and back-illuminated sensor[6].

In general, the tool used to estimate the spatial resolution is the MTF (Modulation Transfer Function) defined in Fourier space. It is used to evaluate the resolution performance of a device as a signal passes through it. The MTF curves represent the loss of contrast as a function of spatial frequency. Imaging relies largely on the notion of spatial resolution as well as on criteria in terms of contrast at a certain spatial frequency, often the MTF for $10 \%$. In this study, the spatial resolution is defined as the period of a $10 \%$ attenuated sinusoidal signal, SR $10 \%$, with (1).

$$
\mathrm{SR} 10 \%=\frac{1}{f(M T F=10 \%)}
$$

The experimental measurements provided MTF curves for the different detection elements. The corresponding spatial resolution values are listed in Table I. The spatial resolution of the HyperPrime lens was limiting, so a replacement of the lens would improve the total spatial resolution of the optical system.

Several lens were considered and compared based on their MTF curves provided by the manufacturers. The most suitable lens for our study with the lowest spatial resolution is the Sigma $105 \mathrm{~mm}$ lens [7]. After experimental measurements in the laboratory, its spatial resolution was measured at $7 \mu \mathrm{m}$ (Table I).
TABLE I

MTF AND SPATIAL RESOLUTION VALUES OF THE DIFFERENT DETECTION ELEMENTS OF THE TOMOGRAPH

\begin{tabular}{ccc}
\hline \hline Detection element & $\begin{array}{c}\text { MTF }(10 \%) \\
\text { In sensor plane }\end{array}$ & $\begin{array}{c}\text { SR10\% } \\
\text { In sensor plane }\end{array}$ \\
\hline Zyla camera sensor & $111 \pm 12$ cycle. $\mathrm{mm}^{-1}$ & $9 \pm 1 \mu \mathrm{m}$ \\
Lens HyperPrime & $83 \pm 18$ cycle. $\mathrm{mm}^{-1}$ & $12 \pm 3 \mu \mathrm{m}$ \\
$\begin{array}{c}\text { Optic global } \\
\text { (Zyla }+ \text { HyperPrime) }\end{array}$ & $59 \pm 2$ cycle. $\mathrm{mm}^{-1}$ & $17 \pm 1 \mu \mathrm{m}$ \\
Marana camera sensor & $39 \pm 4$ cycle. $\mathrm{mm}^{-1}$ & $26 \pm 3 \mu \mathrm{m}$ \\
Lens Sigma $105 \mathrm{~mm}$ & $140 \pm 30$ cycle. $\mathrm{mm}^{-1}$ & $7 \pm 1 \mu \mathrm{m}$ \\
\hline \hline
\end{tabular}

\section{STEP 2: SCINTILLATOR SELECTION}

The scintillator currently used with the SATURNE accelerator is a Gadox screen (Gadolinium oxysulfide $\left.\left(\mathrm{Gd}_{2} \mathrm{O}_{2} \mathrm{~S}\right)\right)$. A state of the art of high-energy and high-resolution tomography systems in use around the world has identified a high-energy tomography system with the highest resolution published so far. It is used in the United States to monitor the performance of aging weapons and is called CoLOSSIS (Confined Large Optical Scintillator Screen and Imaging System). This system is developed by the Lawrence Livermore National Laboratory (LLNL) [8] [9]. The real technological achievement of this system is the CoLOSSIS detector. It is composed of a transparent ceramic GLO $\left(\mathrm{GD}_{0.3} \mathrm{Lu}_{1.6} \mathrm{Eu}_{0.1} \mathrm{O}_{3}\right)$ scintillator [10]. This scintillator is specially designed for high energy and high resolution. Its characteristics are listed in Table II and compared with those of other scintillators typically used in imaging, including the one currently used by the LMN (Gadox).

The GLO is very thin $(1.58 \mathrm{~mm})$ but is much denser than typical imaging scintillators, with a density of $9.1 \mathrm{~g} . \mathrm{cm}^{-3}$. Therefore, it has a higher photon stopping power than Gadox for an equivalent thickness. In addition, it has a high light output and can be produced in large dimensions, up to $30 \mathrm{~cm}$ x $30 \mathrm{~cm}$. However, its most interesting feature is its high spatial resolution. The GLO scintillator has a 10\% spatial resolution of $150 \mu \mathrm{m}$.

TABLE II

COMPARISON OF THE CHARACTERISTICS OF DIFFERENT SCINTILLATORS TYPICALLY USED IN IMAGING AND THE GLO SCINTILLATOR

\begin{tabular}{|c|c|c|}
\hline Scintillator & $\begin{array}{l}\text { Volumic mass } \rho \\
\qquad\left(\mathrm{g} / \mathrm{cm}^{3}\right)\end{array}$ & $\begin{array}{c}\text { Photon yield } \\
(\mathrm{ph} / \mathrm{MeV})\end{array}$ \\
\hline $\begin{array}{c}G L O \\
\left(G D_{0.3} L u_{1.6} 1 E u_{0.1} O_{3}\right)\end{array}$ & 9.1 & 5500 \\
\hline$C s I(T I)$ & 4.5 & 6500 \\
\hline $\mathrm{CdWO}_{4}$ & 7.9 & 2800 \\
\hline $\begin{array}{l}\text { Gadox }(G O S \\
\left(\mathrm{Gd}_{2} \mathrm{O}_{2} \mathrm{~S}: \mathrm{Tb}\right)\end{array}$ & 4.5 & 55000 \\
\hline
\end{tabular}


In view of all its characteristics, the GLO scintillator seems to be the most appropriate for the design of our detector. It offers the best compromise between stopping power, light output and spatial resolution.

\section{STEP 3: OPTIC CONFIGURATION}

The aim of this section is to numerically determine which configuration of the detection optics is the best to achieve the smallest possible detector total spatial resolution in the object plane.

Different configurations were tested by changing the cameras, the lenses (using the performance tests from Step 1, see section II) and the camera configuration. In fact, we can use the current configuration of the tomograph used by the LMN with a single out-of-field camera imaging the whole scintillator (Fig. 2), or a coupling of four cameras, each imaging a quarter of the scintillator, which would allow a better spatial resolution. The geometries of these configurations are shown in Fig. 3. The four studied configurations are:

- $\quad$ one Zyla camera with a HyperPrime lens (current tomograph configuration),

- $\quad$ one Zyla camera with a Sigma $105 \mathrm{~mm}$ lens,

- a coupling of four Zyla cameras with Sigma $105 \mathrm{~mm}$ lenses,

- $\quad$ one Marana camera with a Sigma $105 \mathrm{~mm}$ lens.

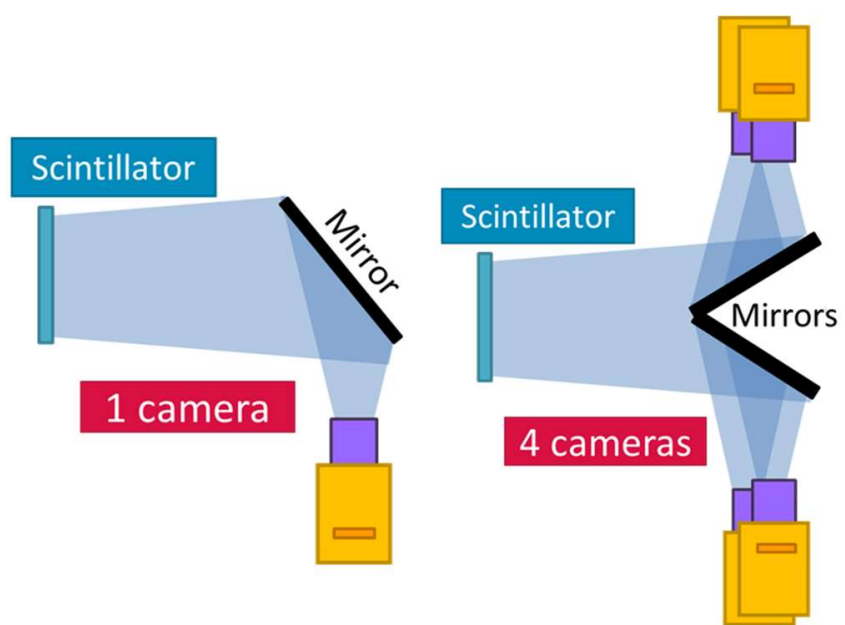

Fig. 3. Diagrams of the proposed camera configurations: either a single camera imaging the whole scintillator or four cameras each imaging a quarter of the scintillator.

To select the best configuration, a calculation of the detector total spatial resolution in the object plane is performed by using the MTFs obtained in the previous steps (MTF Zyla camera, MTF GLO scintillator, MTF Sigma lens, etc. ) according to the magnification, M, applied to the system which is defined with (2).

$$
M=\frac{\text { Source }- \text { detector distance }}{\text { Source-object distance }}=\frac{S D}{S O}
$$

The use of MTFs is advantageous because the total detector MTF in the object plane, and therefore the associated spatial resolution, can be obtained by multiplying the MTF of the different elements in the object plane.

The total detector spatial resolution values in the object plane for a magnification of 1.3 and 2 are listed in Table III.

TABLE III

VALUES OF THE SPATIAL RESOLUTION TOTAL OF THE DETECTOR IN THE OBJECT PLANE FOR DIFFERENT MAGNIFICATIONS FOR THE FOUR PROPOSED OPTICAL

\begin{tabular}{ccc}
\multicolumn{3}{c}{ CONFIGURATIONS } \\
\hline \hline Optic configuration & $\begin{array}{c}\mathrm{M}=1.3 \\
\text { SR10\%Total detector }\end{array}$ & $\begin{array}{c}\mathrm{M}=2 \\
\text { SR } 10 \% \text { Total detector }\end{array}$ \\
\hline 1 Zyla + HyperPrime & $417 \pm 109 \mu \mathrm{m}$ & $395 \pm 103 \mu \mathrm{m}$ \\
1 Zyla + Sigma & $341 \pm 61 \mu \mathrm{m}$ & $375 \pm 68 \mu \mathrm{m}$ \\
4 Zyla + Sigma & $278 \pm 41 \mu \mathrm{m}$ & $300 \pm 45 \mu \mathrm{m}$ \\
1 Marana + Sigma & $358 \pm 70 \mu \mathrm{m}$ & $395 \pm 77 \mu \mathrm{m}$ \\
\hline \hline
\end{tabular}

The configuration with the coupling of four Zyla cameras and Sigma lenses gives the best detector total spatial resolution. It allows a spatial resolution at $10 \%$ of approximately $300 \mu \mathrm{m}$ for a magnification of 1.3 .

This study also showed that as the magnification increases, the detector total spatial resolution in the object plane deteriorates, so low magnifications should be chosen. The envisaged magnification will therefore be between 1.1 and 1.3

\section{CONCLUSIONS}

All these steps enabled to design a detector to obtain the best total spatial resolution of the system. The final elements composing the dimensioned detector are:

- a GLO scintillator in transparent ceramic of dimension $30 \mathrm{~cm} \times 30 \mathrm{~cm}$,

- a camera configuration with the coupling of four Zyla cameras, coupled with four Sigma $105 \mathrm{~mm}$ lenses,

- $\quad$ a magnification between 1.1 and 1.3.

Through all these steps, the detector dimensioned is expected with a spatial resolution about $300 \mu \mathrm{m}$ for a $10 \%$ contrast without taking into account the contribution of the scattered radiation from the scanned object.

Indeed, complementary investigation is in progress to complete this study by taking into account the amount and spread of radiation scattered by the object using Monte-Carlo simulations and Build-Up factor calculations. Depending on the distance between the object and the source, the radiation scattered by the object will not be detected in the same way due to the scattering angles and attenuation. This study will therefore determine an optimum between the influence of scattered radiation and the overall spatial resolution of the system.

One of the perspectives of this study is the implementation of blur and scattered radiation correction methods on the images from deconvolution in order to further reduce the spatial resolution. This correction could be done with the 
deconvolution of the projections from the global PSF of the system or via the implementation of neural networks.

\section{REFERENCES}

[1] N. Estre, D. Eck, J.-L. Pettier, E. Payan, C. Roure, and E. Simon, "High-energy X-ray imaging applied to non destructive characterization of large nuclear waste drums," in 2013 3rd International Conference on Advancements in Nuclear Instrumentation, Measurement Methods and their Applications (ANIMMA), 2013, pp. 1-6. doi: 10.1109/ANIMMA.2013.6727987.

[2] Detection Technology, "X-Ray Line Detectors - Detection

Technology." https://www.deetee.com/x-ray-line-detectors/ (accessed Jul. 16, 2021).

[3] PerkinElmer, "Flat Panel X-ray Detectors - PerkinElmer." https://www.perkinelmer.com/PDFs/downloads/SPC-NDT-Detectors012163A_01.pdf (accessed Jul. 16, 2021).

[4] “ANDOR : Zyla 5.5 camera specifications," 2019. https://andor.oxinst.com/products/scmos-camera-series/zyla-5-5scmos (accessed Jan. 21, 2019).

[5] "SLR Magic HyperPrime 25 mm lens specifications," 2020. http://www.slrmagic.info/product/HyperPrime+CINE+III+2595MFT (accessed Sep. 30, 2020).

[6] “ANDOR : Marana camera specifications," 2020. https://andor.oxinst.com/products/scmos-camera-series/marana-scmos (accessed Sep. 30, 2020).

[7] "Sigma $105 \mathrm{~mm} \mathrm{f} / 1.4$ DG HSM Art lens specifications," 2020. https:/www.sigma-photo.fr/objectifs-photo/310-105mm-f14-dg-hsmart.html (accessed Sep. 30, 2020).

[8] A. Heller, "A cat scanner for nuclear weapon," Sci. Technol. Rev., p. 28, Aug. 2009.

[9] Lawrence Livermore National Laboratory (LLNL), "Scientists develop new CT scanner to image nuclear weapon components," Jun. 08, 2020. https://www.llnl.gov/news/scientists-develop-new-ctscanner-image-nuclear-weapon-components

[10] N. Cherepy, "MeV Radiography with GLO Transparent Ceramic Scintillator," p. 15, 2016. 